\title{
Tunable microwave phase shifter based on silicon-on-insulator microring resonator
}

Pu, Minhao; Liu, Liu; Xue, Weiqi; Ding, Yunhong; Frandsen, Lars Hagedorn; Ou, Haiyan; Yvind, Kresten; Hvam, Jørn Märcher

Published in:

I E E E Photonics Technology Letters

Link to article, DOI:

10.1109/LPT.2010.2046725

Publication date:

2010

Document Version

Publisher's PDF, also known as Version of record

Link back to DTU Orbit

Citation (APA):

Pu, M., Liu, L., Xue, W., Ding, Y., Frandsen, L. H., Ou, H., Yvind, K., \& Hvam, J. M. (2010). Tunable microwave phase shifter based on silicon-on-insulator microring resonator. I E E Photonics Technology Letters, 22(12), 869-871. https://doi.org/10.1109/LPT.2010.2046725

\section{General rights}

Copyright and moral rights for the publications made accessible in the public portal are retained by the authors and/or other copyright owners and it is a condition of accessing publications that users recognise and abide by the legal requirements associated with these rights.

- Users may download and print one copy of any publication from the public portal for the purpose of private study or research.

- You may not further distribute the material or use it for any profit-making activity or commercial gain

- You may freely distribute the URL identifying the publication in the public portal 


\title{
Tunable Microwave Phase Shifter Based on Silicon-on-Insulator Microring Resonator
}

\author{
Minhao Pu, Liu Liu, Weiqi Xue, Yunhong Ding, Lars H. Frandsen, Haiyan Ou, Kresten Yvind, and Jørn M. Hvam
}

\begin{abstract}
We demonstrate microwave phase shifters based on electrically tunable silicon-on-insulator microring resonators (MRRs). MRRs with different quality factors are fabricated and tested. A continuously tunable phase shift of up to $336^{\circ}$ at a microwave frequency of $40 \mathrm{GHz}$ is obtained using a high-quality-factor ( 28000) MRR with only $1.6-\mathrm{mW}$ power consumption. A quasi-linear phase shift in the range of $0^{\circ}-204^{\circ}$ at $40 \mathrm{GHz}$ with a radio-frequency power variation less than $1.3 \mathrm{~dB}$ is also achieved by using a lower-quality-factor MRR.
\end{abstract}

Index Terms-Integrated optics devices, microwave photonics, radio-frequency (RF) photonics, silicon microring resonator (MRR).

\section{INTRODUCTION}

$\mathbf{M}$ ICROWAVE photonics for processing microwave and millimeter-wave signals in the optical domain has lately received increasing interest [1]. Photonic components, providing compact size, large bandwidth, fast tunability, immunity to electromagnetic interference, and low weight, have been widely demonstrated in microwave systems. Microwave phase shifters are key components in many microwave applications, such as phased-array antennas [2] and microwave filters [3]. So far, several schemes for phase shifting, including wavelength conversion [4], stimulated Brillouin scattering [5], slow-light effects in semiconductor devices [6], and band-edge effects in photonic band-gap devices [7], have been reported. Recently, silicon-on-insulator (SOI) microring resonators (MRRs) were also used to implement phase shifters realizing a $0^{\circ}-260^{\circ}$ shifting range with thermo-optical tuning from a high-power control light of at least $40 \mathrm{~mW}$ [8]. In this letter, we demonstrate an electrically tunable phase shifter based on a high-quality-factor (high- $Q$ ) SOI MRR with a larger phase-shifting range of $0^{\circ}-336^{\circ}$ and a much lower power consumption of $1.6 \mathrm{~mW}$. We also demonstrate a phase shifter with a quasi-linear tuning of $0^{\circ}-204^{\circ}$ and less than $1.3-\mathrm{dB}$

Manuscript received February 05, 2010; revised March 16, 2010; accepted March 20, 2010. Date of publication April 08, 2010; date of current version May 21,2010 . This work was supported by the NANO.COM and GOSPEL projects at DTU Fotonik.

M. Pu, L. Liu, W. Xue, H. Ou, K. Yvind, and J. M. Hvam are with DTU Fotonik, Department of Photonics Engineering, Technical University of Denmark, 2800 Kgs. Lyngby, Denmark (e-mail: mipu@ fotonik.dtu.dk).

Y. Ding is with DTU Fotonik, Department of Photonics Engineering, Technical University of Denmark, 2800 Kgs. Lyngby, Denmark, and also with Huazhong University of Science and Technology, Wuhan National Laboratory for Optoelectronics, 430074 Wuhan, China.

L. H. Frandsen is with NKT Photonics, 3460 Birkerød, Denmark.

Color versions of one or more of the figures in this letter are available online at http://ieeexplore.ieee.org.

Digital Object Identifier 10.1109/LPT.2010.2046725

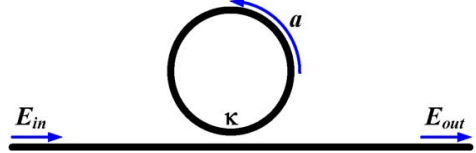

Fig. 1. Schematic of an all-pass MRR.
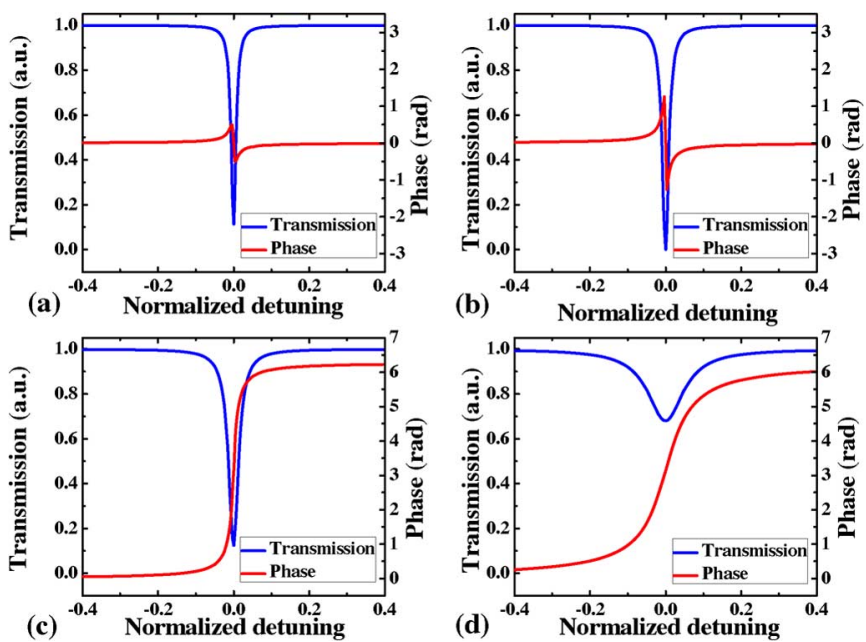

Fig. 2. Transmission and phase shift of the through port for the MRR under different coupling conditions: (a) under-coupling $\kappa^{2}=0.005$, (b) critical-coupling $\kappa^{2}=1-a^{2}=0.01$, (c) over-coupling with $\kappa^{2}=0.02$, and (d) $\kappa^{2}=$ 0.1 . Here, $a^{2}$ is always assumed to be 0.995 .

radio-frequency (RF) power variation employing a low- $Q$ MRR. The tuning operations of our devices are performed by electrical current through integrated heaters, instead of optical power adopted in [8]. This would be a much more practical implementation of such a phase shifter in real applications.

\section{PRINCIPLE}

Fig. 1 shows the schematic drawing of an all-pass MRR, where $\kappa$ and $a$ are amplitude coupling coefficient of the coupling region and amplitude transmission coefficient for a round-trip along the ring, respectively. The transmittance of the complex field at the through port can be expressed as [9]

$$
\frac{E_{\text {out }}}{E_{\text {in }}}=\frac{r-a e^{-j \varphi}}{1-a r e^{-j \varphi}}
$$

where $\varphi$ is the round-trip phase change of the ring and $r$ is amplitude transmission coefficient which satisfies the relation $r^{2}+$ $\kappa^{2}=1$. Fig. 2 shows the transmission and phase at the through port with different coupling coefficients $\kappa$. At the under-coupling and critical-coupling conditions as shown in Figs. 2(a) and (b), respectively, the phase cannot change by more than $\pi$. 


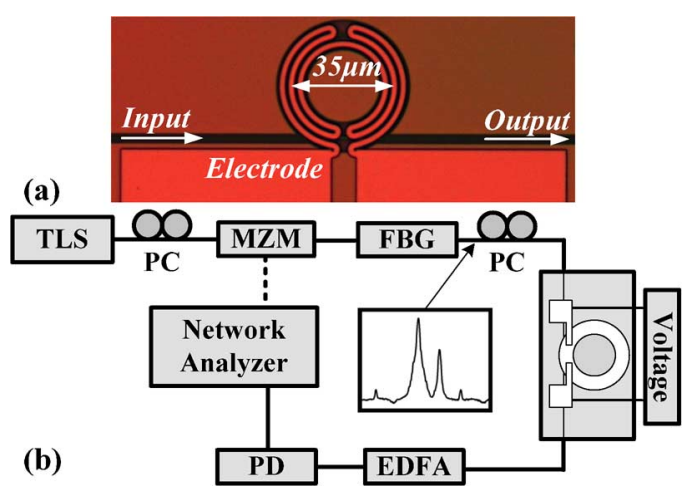

Fig. 3. (a) Optical microscope picture of the fabricated MRR with microheater. (b) Experimental setup for phase-shift measurements. Inset shows the generated microwave signal after the FBG notch filter.

Whereas, at the over-coupling conditions, as shown in Figs. 2(c) and (d), the phases experience a full $2 \pi$ shift. If, in this case, an optical signal carrying a microwave signal with two peaks of the desired frequency spacing is input to the MRR, the phase difference of the two peaks of the transmitted field can be tuned by changing the resonance frequency, and thus realizing a microwave phase shift. Therefore, a tunable MRR working in the over-coupling regime is preferred in order to make a microwave phase shifter with large and continuous tuning range.

\section{FABRICATION}

The tunable MRR was fabricated in an SOI wafer with a top silicon thickness of $250 \mathrm{~nm}$ and a $3-\mu \mathrm{m}$ buried silicon dioxide layer. Electron-beam (EB) lithography and inductively coupled plasma reactive ion etching were used to define the microring structure. Then a 550-nm-thick benzocyclobuten (BCB) top cladding was spin-coated and subsequently hard-cured. After that, 400-nm ZEP520A resist and EB lithography were employed again to define the pattern of the microheater. Evaporation and lift-off techniques were used as the last steps to form 100-nm-thick titanium heaters together with contact pads. Fig. 3(a) shows the optical microscope picture of the fabricated device. The waveguide width is $450 \mathrm{~nm}$ and the diameter of the MRR is $35 \mu \mathrm{m}$ which corresponds to a free-spectral range (FSR) of $640 \mathrm{GHz}$. At both ends of the device, the waveguide is tapered from $450 \mathrm{~nm}$ to $4 \mu \mathrm{m}$ to expand the guided mode for more efficient fiber-to-chip coupling. The insertion loss of the device is $\sim 15 \mathrm{~dB}$.

\section{EXPERIMENT SETUP}

The experimental setup used to measure the fabricated device is shown in Fig. 3(b). Light from a tunable laser source (TLS) was modulated through a Mach-Zehnder modulator (MZM) by a microwave signal from the network analyzer. A fiber Bragg grating (FBG) notch filter was used to filter out one sideband of the modulated signal [10]. After that, the optical signal, with the envelope modulated at the microwave frequency in the time domain [i.e., with two peaks of the desired frequency spacing in the spectral domain as shown in the inset of Fig. 3(b)] was generated and sent into the fabricated sample. The polarization of the input light was adjusted to the quasi-transverse-electric
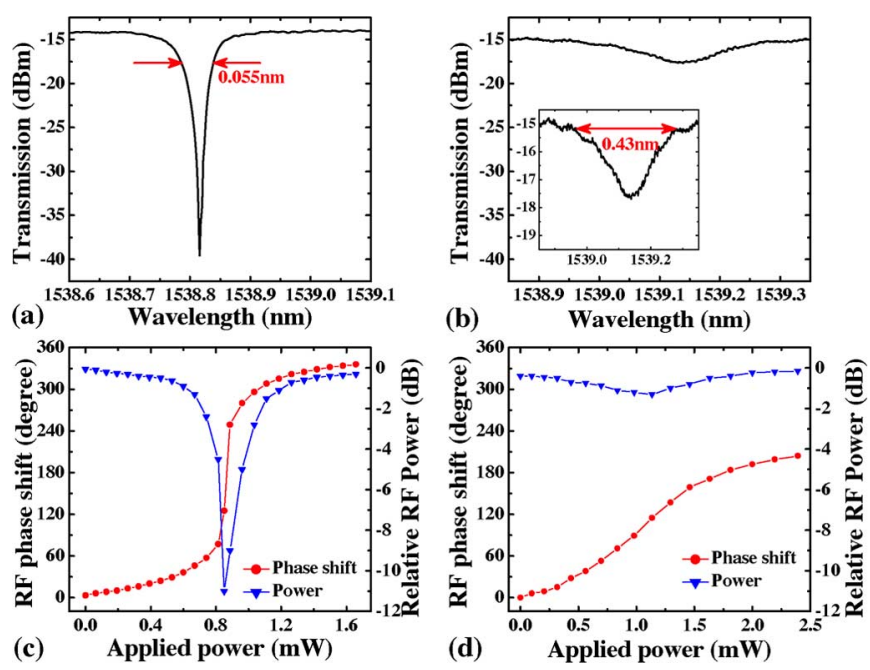

Fig. 4. Transmission spectrum of the MRRs with a coupling gap of (a) $225 \mathrm{~nm}$ and (b) $100 \mathrm{~nm}$ for the TE mode. Inset is the zoomed view for (b). Measured RF phase shift and relative RF power versus the applied power to the microheater on the MRR with a coupling gap of (c) $225 \mathrm{~nm}$ and (d) $100 \mathrm{~nm}$.

(quasi-TE) mode with a fiber polarization controller (PC). By applying a voltage to the microheater, the resonance frequency of the MRR can be tuned with respect to one of the peaks of the optical signal to change the phase difference between the two peaks. Amplified by an erbium-doped fiber amplifier (EDFA), the output signal was detected by a photodetector (PD), and converted to the microwave signal. Then the network analyzer was used to extract the information of phase and power changes of the microwave signal.

\section{EXPERIMENTAL RESULTS AND DISCUSSION}

An MRR with a ring-to-waveguide gap (coupling gap) of $225 \mathrm{~nm}$ was first tested. Fig. 4(a) shows the transmission spectrum of the MRR for quasi-TE mode. The MRR has an extinction ratio of $\sim 26 \mathrm{~dB}$ and $0.055-\mathrm{nm} 3-\mathrm{dB}$ bandwidth $(Q \sim$ 28000 ), slightly above the critical coupling condition. Fig. 4(c) shows the measured RF phase shift and RF power variation as a function of applied electrical power to the microheater. A continuously tunable RF phase shift is demonstrated, and the maximum RF phase shift of $336^{\circ}$ is achieved. However, the large RF power variation (about $11 \mathrm{~dB}$ ) due to the high extinction ratio of the MRR hampers the application as an RF phase shifter. This problem can be resolved by using a low- $Q$ MRR with a higher coupling coefficient (narrower coupling gap), which will give a lower extinction ratio. An MRR with a coupling gap of $100 \mathrm{~nm}$ and $2.7-\mathrm{dB}$ extinction ratio was then tested. The transmission spectrum and RF phase shift and RF power variation versus applied heating power are shown in Figs. 4(b) and (d), respectively. The RF power variation is about $1.3 \mathrm{~dB}$, which is $\sim 9.7 \mathrm{~dB}$ smaller than that of the high- $Q$ MRR. Although the maximum achievable RF phase shift $\left(\sim 204^{\circ}\right)$ is smaller due to the wide resonant bandwidth, the RF phase shift is more linear as compared to the high- $Q$ MRR.

We investigated the maximum RF phase shift in the proposed devices with different coupling coefficients, as shown in Fig. 5(a). As expected, the maximum RF phase shift increases 


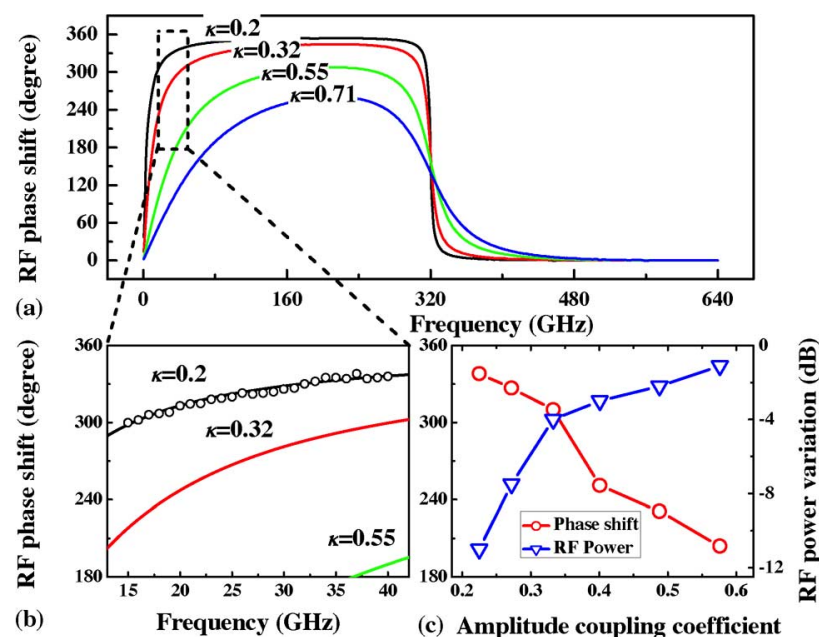

Fig. 5. (a) Calculated maximum RF phase shift versus the microwave frequencies for different coupling coefficients. (b) Zoomed view of (a) within the frequencies from 13 to $42 \mathrm{GHz}$. Open circles are the measured maximum RF phase shift versus the microwave frequencies with the MRR of $28000 Q$-factor. (c) Measured maximum RF phase shift and RF power variation for MRRs with different ring-to-waveguide gaps at a frequency of $40 \mathrm{GHz}$.

as the RF frequency increases, and then drops back to zero when the RF frequency approaches the FSR $(640 \mathrm{GHz})$. The MRR with a lower coupling coefficient offers a larger tuning range for the RF phase. The measured maximum RF phase shifts for different microwave frequencies modulated on the optical beam for the high- $Q$ MRR are shown as the open circles in Fig. 5(b), which shows a good agreement with the theoretical prediction. Fig. 5(c) shows the measured RF power variation and maximum RF phase shift for the MRRs with different amplitude coupling coefficients (different coupling gaps). As the coupling gap increases, the coupling coefficient $\kappa$ decreases which leads to a larger $Q$-factor and a higher extinction ratio. One finds that the MRR with higher $Q$ (lower $\kappa$ ) provides a larger RF phase shift. However, the lower- $Q$ MRR gives a smaller RF power variation at the expense of reduced maximum $\mathrm{RF}$ phase shift. Since the RF power variation is a vital factor in many microwave applications, the low- $Q$ MRR with small $\mathrm{RF}$ power variation and good phase-shift linearity would be a more practical option for the real applications. In this case, two cascaded MRRs are necessary to achieve a full $360^{\circ} \mathrm{RF}$ phase shift.

Compared to the device in [8] where the MRR was tuned by a strong optical power, our device is more energy efficient and offers larger RF phase shift. Only a small electrical power of $0.9 \mathrm{~mW}$ is needed to obtain $\sim 260^{\circ}$ ( $\left.\sim 4.6 \mathrm{rad}\right) \mathrm{RF}$ phase shift which is $2.25 \%$ of the optical power in the device in [8].

In addition, our device can be easily cascaded and controlled independently to realize a linear and full $2 \pi$ RF phase shift. Fig. 6 shows the calculated transmission and phase at the through port of cascaded two MRRs with different resonance offsets $\left(\omega_{\mathrm{MRR} 2}-\omega_{\mathrm{MRR} 1}\right)$ between the two MRRs. A total phase change of $720^{\circ}$ can be achieved for this configuration. As shown in Fig. 6, the transmission spectrum and the phase curve can be altered by offsetting the resonances for the two MRRs. Therefore, the resonance offset can be tuned to a desired value (e.g., $3 \mathrm{GHz}$ in this case) to obtain a wide notch bandwidth and
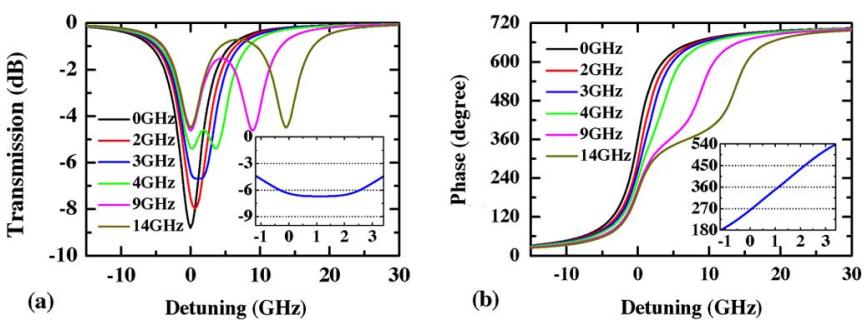

Fig. 6. (a) Calculated optical transmission and (b) phase as a function of the detuning $\left(\omega-\omega_{\text {MRR1 }}\right)$ for the cascaded MRRs with different resonance offsets $\left(\omega_{\text {MRR2 }}-\omega_{\text {MRR1 }}\right)$. Insets are zoomed views for the cascaded MRRs with $3-\mathrm{GHz}$ resonance offset for a detuning range from -1.2 to $3.4 \mathrm{GHz}$. Here, $\kappa^{2}, a^{2}$ are always assumed to be 0.04 and 0.995 , respectively.

a flattened notch bottom for the transmission and a linear shift for the phase in a certain detuning range, as shown in the insets in Fig. 6. If the microwave phase shifter is operated within this detuning regime, one can realize a linear $2 \pi$ RF phase shift with mimimal RF power variation since the RF power follows the optical power.

\section{CONCLUSION}

We have introduced microwave phase shifters based on electrically tunable SOI MRRs. A phase-shifting range of $0^{\circ}-336^{\circ}$ has been achieved at a microwave frequency of $40 \mathrm{GHz}$ with an MRR of $Q=28000$. A smooth phase shift up to $204^{\circ}$ has also been demonstrated with less than 1.3-dB RF power variation using a lower- $Q$ MRR. It is feasible to realize a continuously tunable $360^{\circ}$ microwave phase shifter with small power consumption by cascading two such MRRs.

\section{REFERENCES}

[1] J. Capmany and D. Novak, "Microwave photonics combines two worlds," Nat. Photon., vol. 1, pp. 319-330, Jun. 2007.

[2] S. Tonda-Goldstein, D. Dolfi, A. Monsterleet, S. Formont, J. Chazelas, and J. P. Huignard, "Optical signal processing in radar systems," IEEE Trans. Microw. Theory Tech., vol. 54, no. 2, pt. 2, pp. 847-853, Feb. 2006.

[3] J. Capmany, B. Ortega, D. Pastor, and S. Sales, "Discrete-time optical processing of microwave signals," J. Lightw. Technol., vol. 23, no. 2, pp. 702-723, Feb. 2005.

[4] M. Fisher and S. Chuang, "A microwave photonic phase-shifter based on wavelength conversion in a DFB laser," IEEE Photon. Technol. Lett., vol. 18, no. 16, pp. 1714-1716, Aug. 15, 2006.

[5] A. Loayssa and F. J. Lahoz, "Broad-band RF photonic phase shifter based on stimulated Brillouin scattering and single-sideband modulation," IEEE Photon. Technol. Lett., vol. 18, no. 1, pp. 208-210, Jan. 1, 2006.

[6] W. Xue, S. Sales, J. Capmany, and J. Mørk, "Microwave phase shifter with controllable power response based on slow- and fast-light effects in semiconductor optical amplifiers," Opt. Lett., vol. 34, pp. 929-931, Apr. 2009.

[7] M. Povinelli, S. Johnson, and J. Joannopoulos, "Slow-light, band-edge waveguides for tunable time delays," Opt. Express, vol. 13, pp. 7145-7159, 2005.

[8] Q. Chang, Q. Li, Z. Zhang, M. Qiu, T. Ye, and Y. Su, "A tunable broadband photonic rf phase shifter based on a silicon microring resonator,' IEEE Photon. Technol. Lett., vol. 21, no. 1, pp. 60-62, Jan. 1, 2009.

[9] J. Heebner, V. Wong, A. Schweinsberg, R. Boyd, and D. Jackson, "Optical transmission characteristics of fiber ring resonators," IEEE J. Quantum Electron., vol. 40, no. 6, pp. 726-730, Jun. 2004.

[10] G. Qi, J. Yao, J. Seregelyi, S. Paquet, and C. Belisle, "Generation and distribution of a wide-band continuously tunable millimeter-wave signal with an optical external modulation technique," IEEE Trans. Microw. Theory Tech., vol. 53, no. 10, pp. 3090-3097, Oct. 2005. 